\title{
Noise Analysis of Magnetic Sensors Using Allan Variance
}

\author{
K. Draganová*, F. Kmec, J. Blažek, D. PrasličKa, J. Hudák, M. LašŠÁK \\ Department of Aviation Technical Studies, Faculty of Aeronautics, Technical University of Kosice, \\ Rampová 7, 04121 Košice, Slovakia
}

\begin{abstract}
The article deals with the noise analysis of magnetic sensors using Allan variance. In comparison with the standard variance, based on the variations around the average value, Allan variance provides a measure of the behavior of the variability of a quantity as it is averaged over different measurement time periods, which results in a better convergence and the possibility to distinguish types of noise directly. A significant advantage of this method is that there is no need of any transformations and according to the IEEE recommendation the Allan variance approach is the preferred method for stochastic error identification and the noise type determination in inertial sensors (accelerometers and gyroscopes). Considering that magnetic sensors become even more often a part of inertial measurements units, the goal of this article is to prove that the method using the Allan variance analysis can be very successfully applied also for noise analysis in magnetic sensors.
\end{abstract}

DOI: 10.12693/APhysPolA.126.394

PACS: 07.50.Hp

\section{Introduction}

An important factor that affects the precision of the sensor measurement is noise. Magnetic sensor noise has been investigated by many authors from different points of view [1]. One of perspective methods is a technique based on Allan Variance (AV) calculation [2, 3]. The goal of this article is to prove that this method is convenient also for magnetic sensor noise type determination. And forasmuch as it was proved that sensor noise depends mostly on the core material and its geometry aspect, measurements for theory confirmation were performed on two different core types.

\section{Theory}

The AV of a time series of data is a characterization of the noise and other processes in the data as a function of averaging time, without the need of any transformation [4]. This statistical metrological method is a measure of the fractional frequency fluctuations and can be used for a noise type determination in the time domain and can be calculated from the measured output signal of a magnetic sensor with $N$ samples, after that it is divided into clusters $k$. Considering $m$ samples in one cluster we get $K=N / m$ clusters:

$$
\underbrace{a_{(1)}, a_{(2)}, \ldots a_{(m)}}_{k=1} \cdots \underbrace{a_{(N-m)}, a_{(N-m+1)}, \ldots a_{(N)}}_{k=K},
$$

where $K$ indicates number of clusters (intervals) and $m$ designates number of samples in the cluster. For each cluster its average value $\bar{a}[k]$ has to be determined:

$$
\begin{aligned}
& \bar{a}[k]=\frac{1}{m} \sum_{i=1}^{m} a((k-1) m+i) ; \\
& k=1,2, \ldots K .
\end{aligned}
$$

In consequence we can write relationship for the AV:

\footnotetext{
*corresponding author; e-mail: katarina.draganova@tuke.sk
}

$$
\begin{aligned}
& \sigma_{A}^{2}(\tau)=\frac{1}{2(K-1)} \sum_{k=1}^{K-1}(\bar{a}[k+1]-\bar{a}[k])^{2} ; \\
& k=1 \ldots K-1,
\end{aligned}
$$

where $\sigma_{A}$ represents Allan variance with non-overlapping samples and $\tau$ is an averaging time. Considering sampling frequency $f$ for the averaging time $\tau$ we can write:

$$
\tau=\frac{m}{f} \text {. }
$$

The noise parameter $Q$ for the specific noise type can be expressed as:

$$
Q=\sigma_{A}(\tau) \sqrt{\tau}
$$

Based on the formulas (1)-(3) it is obvious that the relative error $\delta[\%]$ is dependent on the number of clusters $K$, according to the relationship:

$$
\delta=\frac{100}{\sqrt{2(K-1)}} .
$$

Increasing number of clusters causes the increase of error and the precision of the AV estimation is decreasing.

Application of this calculation method allows us to determine the prevailing noise type for the averaging period. For this purpose the $\sigma_{A}$ dependence on the averaging time $\tau$ is determined.

There is also an evident dependence between the log$\log$ slopes in plots of square root of Allan variance versus averaging time and in plots of power spectral density versus frequency. Table summarizes the log-log slopes of the various noise processes in a magnetic sensor using AV method and also the corresponding PSD log-log slopes.

Slopes in AV and PSD log-log plots for the magnetic sensor data.

\begin{tabular}{c|c|c}
\hline \hline Signal & $\sigma_{A}$ vs. $\tau$ & PSD vs. $f$ \\
\hline Random walk & $+1 / 2$ & -2 \\
\hline Flicker noise & 0 & -1 \\
\hline Random walk or white noise & $-1 / 2$ & 0 \\
\hline White or quantization noise & -1 & +2
\end{tabular}




\section{Experiments and results}

Measurements were performed using developed modular magnetometer with CPLD (Complex Programmable Logical Device) controlled electronics based either on the amorphous ribbons or Co-based magnetic microwires as sensor's sensing elements. Measurements are performed using induction method, based on an exciting coil supplied by the triangular-shaped current and the sensing coil for the induced voltage peaks detection. The measured switching field is proportional to the time at which peaks appear. Sampling frequency during measurement of amorphous ribbons was $1 \mathrm{kHz}$. During the measurement of Co-based magnetic microwires it was $500 \mathrm{~Hz}$.

Magnetometer output data of one magnetometer channel were subsequently processed and $\mathrm{AV}$-equation (3) and PSD were calculated and visualised (Figs. 1-3).

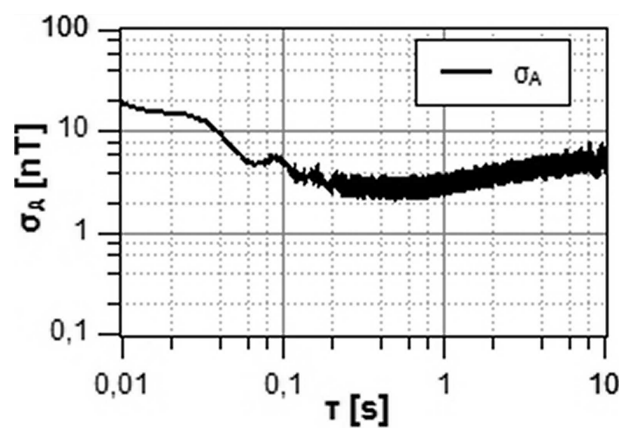

Fig. 1. AV calculated for amorphous ribbon core.

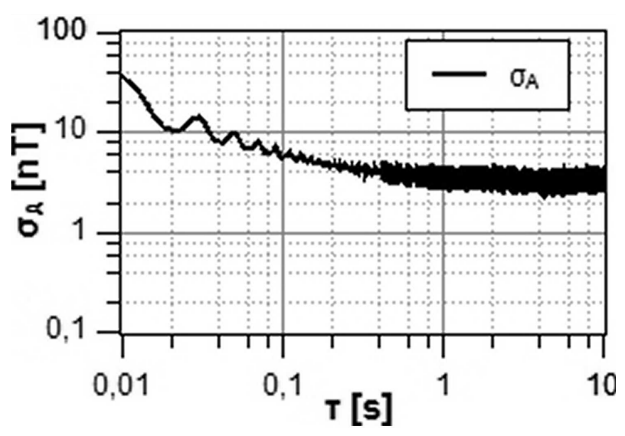

Fig. 2. AV calculated for Co-based magnetic microwire core.

From the calculated characteristics we can see that in case of the amorphous ribbon core (Fig. 1) the random walk or white noise is dominant for lower averaging time and then the trend changes to the random walk due to the inherent instability in the sensor output. The noise type in case of the Co-based core is different and changes from the flicker noise for lower frequencies to the white noise or random walk for frequencies higher than $1 \mathrm{~Hz}$, which confirmed also the PSD vs. frequency graph shown in Fig. 3, determined from the same data series as $\sigma_{A}$ vs. $\tau$ graph shown in Fig. 2. For a better demonstration, a green coloured trend line into the characteristics in Fig. 3 was amended. Co-based core utilization is characterized by a higher noise parameter $\left(36.23 \mathrm{nT} \mathrm{s}^{-1 / 2}\right)$, calculated according to the relationship (3), in comparison to amorphous ribbons where noise parameter was $28.28 \mathrm{nT} \mathrm{s}^{-1 / 2}$.

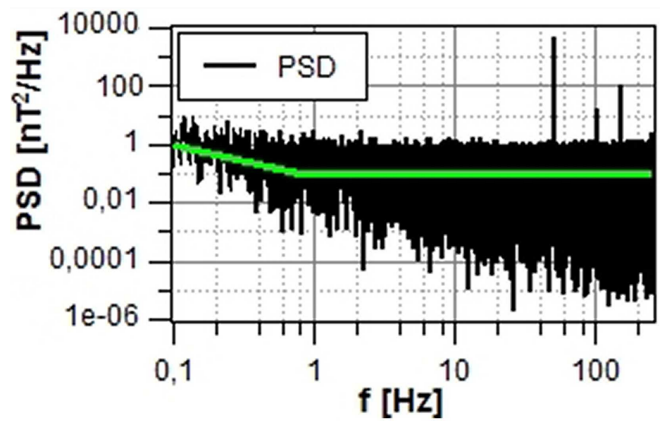

Fig. 3. PSD calculated for Co-based magnetic microwire core.

\section{Conclusions}

Statistical method of noise analysis based on the Allan Variance in comparison to other conventional statistical methods has several significant advantages, there is no need of any transformation, it is convergent for many noise types and according to the IEEE recommendation the AV approach together with the power spectral density approach are the preferred methods for the stochastic error identification of inertial sensors - accelerometers and gyroscopes.

For the verification of the suitability of AVbased methodology, the magnetometers with amorphous ribbon- and Co-based magnetic sensing elements were tested. In both cases a very good convergence was achieved although for AV calculation technique with nonoverlapping samples were used. Allan deviation analysis can also identify and provide excellent differentiation between the particular noise types. Furthermore the AV plots show clearly the time regions over which these noise types are dominant.

\section{Acknowledgments}

This work was supported by the Slovak Research and Development Agency under contract No. APVV 0266-10.

\section{References}

[1] Magnetic Sensors and Magnetometers, Ed. P. Ripka, Artech, Boston 2001, ISBN 1-58053-057-5.

[2] IEEE Std 1554 ${ }^{T M}$-2005: IEEE Recommended Practice for Inertial Sensor Test Equipment, Instrumentation, Data Acquisition, and Analysis, IEEE Aerospace and Electronic System Society, 2005.

[3] M.H. Afzal, http://www.geomatics.ucalgary.ca/ graduatethesesUse of Earth's Magnetic Field for Pedestrian Navigation, Ph.D. Thesis, University of Calgary, Alberta 2011.

[4] D.W. Allan, IEEE Trans. on Instrumentation and Measurement IM-36, 646 (1987). 